\title{
Recomendaciones de la Liga Europea contra el Reumatismo para el manejo de la gota
}

European League Against Rheumatism recommendations for the management of gout

Richette P, y col. Ann Rheum Dis 2017;76:29-42

\section{Introducción}

La gota es un tipo de artritis inflamatoria inducida por el depósito de cristales de urato monosódico (UMS) en el líquido sinovial y en otros tejidos. Constituye un problema médico común, que afecta al menos al $1 \%$ de los hombres en países occidentales. La relación hombres/mujeres es de aproximadamente 7/1 a 9/1, hasta la menopausia en donde la prevalencia de gota aumenta en las mujeres por efecto probablemente hormonal y en asociación con el uso de diuréticos. La hiperuricemia es una condición necesaria para el desarrollo de la gota, pero no causa invariablemente la enfermedad. La hiperuricemia se define como un nivel de ácido úrico (AU) sérico mayor o igual a $6,8 \mathrm{mg} / \mathrm{dl}$, que es el límite de solubilidad del AU a temperatura y $\mathrm{pH}$ fisiológicos. Además del nivel de AU sérico, existen otros predictores del desarrollo de gota que incluyen la hipertensión arterial, el uso de tiazidas y diuréticos de asa, la obesidad y un alto consumo de alcohol, todos los cuales parecen contribuir de manera aditiva al riesgo de desarrollar gota. Los síntomas clásicos de la artritis gotosa son ataques agudos recurrentes, marcadamente dolorosos mono u oligoarticulares, pero que pueden dar una verdadera poliartritis, inclusive crónica, haciendo que el diagnóstico diferencial de la artritis gotosa sea muy amplio. Se debe recordar a los fines diagnósticos que los niveles de AU sérico suelen ser normales durante los ataques de la gota aguda y que el diagnóstico definitivo requiere la identificación directa de cristales de urato monosódico (UMS) a través del estudio del líquido sinovial o del estudio de los tofos ${ }^{1,2}$. Recientemente han sido publicados los "Nuevos Criterios de Clasificación" para la gota que introducen básicamente la utilización de las imágenes para el diagnóstico de esta enfermedad ${ }^{3,4}$
Descripción general de la hipótesis y los métodos utilizados En el presente trabajo se convocó un grupo de trabajo para actualizar las recomendaciones de la EULAR 2006 (por sus siglas en inglés European League Against Rheumatism o Liga Europea contra el Reumatismo) para el tratamiento de la gota, con el objetivo de abordar todos los principios generales y las recomendaciones individuales a través de una revisión sistemática de la literatura y la opinión de expertos y pacientes.

El Grupo de trabajo EULAR comprendía 15 reumatólogos, 1 radiólogo musculoesquelético, 2 médicos generalistas, 1 investigador, 2 pacientes y 3 expertos en epidemiología/metodología, todos provenientes de 12 países europeos. Este grupo de trabajo determinó si las 12 recomendaciones del 2006 debían ser sostenidas, modificadas o abandonadas. Posteriormente, con la ayuda de un experto en metodología de revisión sistemática, se buscó la literatura publicada desde el 1 de enero de 2005 hasta junio de 2013 en las bases de datos MEDLINE, EMBASE y Cochrane. Este proceso incluyó tanto una búsqueda general como búsqueda específica de proposiciones. La calidad de la evidencia y los grados de recomendación se determinaron de acuerdo con los estándares del Centro de Oxford para la Medicina Basada en la Evidencia y GRADE. Finalmente, el grupo de trabajo debatió y evaluó las pruebas, produciendo un conjunto de 14 recomendaciones usando la metodología Delphi.

Los principios generales del abordaje con gota se resumen en la tabla 1. En la tabla 2 se sintetiza el grado de recomendación de los tratamientos disponibles, y en la figura 2, el algoritmo de manejo de la hiperuricemia en pacientes con gota.

Toda persona con gota debe estar plenamente informada sobre la fisiopatología de la enfermedad, la existencia de tratamientos efectivos, las comorbilidades asociadas y los principios en el manejo de ataques agudos y acerca de la posibilidad de eliminación de los cristales de UMS a través de la reducción en los niveles de AU por debajo de un nivel objetivo.

Cada persona con gota debe recibir consejos sobre estilo de vida: disminución de peso en el caso de ser necesario, evitar el consumo de alcohol (especialmente cerveza y licores), bebidas azucaradas, comidas pesadas y la ingesta excesiva de carne y mariscos. Se debe alentar el consumo de productos lácteos bajos en grasa y aconsejar la realización de ejercicio en forma regular.

Todas las personas con gota deben someterse sistemáticamente a pruebas de detección de comorbilidades asociadas y factores de riesgo cardiovascular, incluyendo insuficiencia renal, cardiopatía coronaria, insuficiencia cardíaca, accidente cerebrovascular, enfermedad arterial periférica, obesidad, hiperlipidemia, hipertensión arterial, diabetes y tabaquismo, como parte del manejo general de la gota.

*Adaptado del artículo original. UMS: urato monosódico. AU: ácido úrico. 
Los ataques agudos de gota deben ser tratados tan pronto como sea posible. Los pacientes deben ser informados y educados para automedicarse ante los primeros síntomas de alarma. La elección del fármaco debe basarse en la presencia de contraindicaciones, experiencia previa del paciente con los tratamientos, el tiempo de evolución después del inicio del ataque y el número y tipo de articulaciones comprometidas.

Las opciones recomendadas de primera línea para los ataques agudos son la colchicina (dentro de las 12 horas del inicio del ataque) a una dosis de carga de $1 \mathrm{mg}$ seguida 1 hora más tarde por $0,5 \mathrm{mg}$ el día $1 \mathrm{y} / 0$ un AINE (más inhibidores de la bomba de protones, de ser necesario), corticosteroides orales (30 a $35 \mathrm{mg} /$ día de prednisona 0 equivalente durante 3 a 5 días) 0 artrocentesis e inyección local de corticosteroides. La colchicina y los AINEs deben ser evitados en pacientes con insuficiencia renal grave. La colchicina no debe administrarse a pacientes que reciben inhibidores fuertes de la glicoproteína-P y/o CYP3A4 como la ciclosporina o la claritromicina.

En pacientes con ataques frecuentes y contraindicaciones para recibir colchicina, AINE y corticosteroides (oral e inyectable), se deben considerar los bloqueadores de IL-1 (canakinumab o ankinra) para el tratamiento de los ataques. La presencia de infección es una contraindicación para el uso de bloqueadores de IL-1. El tratamiento hipouricemiante debe ajustarse para alcanzar el objetivo de la uricemia y luego el tratamiento de bloqueo de la IL-1 para el ataque.

La profilaxis contra los ataques debe ser explicada y discutida con el paciente. La profilaxis se recomienda durante los primeros seis meses de tratamiento hipouricemiante. El tratamiento profiláctico recomendado es colchicina, 0,5 a $1 \mathrm{mg} / \mathrm{dí}$, una dosis que debe reducirse en pacientes con insuficiencia renal. En los casos de insuficiencia renal 0 tratamiento con estatinas, los pacientes y los médicos deben ser conscientes de la neurotoxicidad o toxicidad muscular potencial asociadas con el uso profiláctico de colchicina. Debe evitarse la co-prescripción de colchicina con inhibidores fuertes de la glicoproteína-P y/o CYP3A4. Si la colchicina no es tolerada 0 está contraindicada, debe considerarse la profilaxis con AINE a dosis bajas, si no están contraindicados.

El tratamiento hipouricemiante debe ser considerado y discutido con cada paciente con diagnóstico definitivo de gota desde el primer ataque. Está indicado en todos los pacientes con ataques recurrentes, presencia de tofos, artropatía crónica y/0 cálculos renales. El inicio del tratamiento hipouricemiante se recomienda cerca del momento del primer diagnóstico en pacientes que se presentan a una edad temprana ( $<40$ años) 0 con un nivel de ácido úrico (AU) muy alto $(>8.0 \mathrm{mg} / \mathrm{dL}$ ) y/o por la presencia de comorbilidades (insuficiencia renal, hipertensión, cardiopatía isquémica, insuficiencia cardíaca). Los pacientes con gota deben recibir información completa y participar plena y activamente en la toma de decisiones sobre el tratamiento hipouricemiante.

Para los pacientes con tratamiento hipouricemiante, el nivel de AU debe ser monitoreado y mantenido en $<6 \mathrm{mg} / \mathrm{dL}$. Para los pacientes con gota severa (tofos, artropatía crónica, ataques frecuentes), se recomienda un objetivo inferior $(<5 \mathrm{mg} / \mathrm{dL})$ hasta la disolución total del cristal y la resolución de la gota. No se recomienda un nivel de $\mathrm{AU}<3 \mathrm{mg} / \mathrm{dL}$ a largo plazo.

Todos los tratamientos hipouricemiantes deben iniciarse con una dosis baja y luego aumentados hasta alcanzar el objetivo de AU. La meta de $\mathrm{AU}<6 \mathrm{mg} / \mathrm{dL}$ ( $360 \mathrm{mmol} / \mathrm{L})$ debe mantenerse durante toda la vida.

En los pacientes con función renal normal, se recomienda el uso de alopurinol para el tratamiento de primera línea, comenzando con una dosis baja (100 mg / día) y aumentándola en incrementos de $100 \mathrm{mg}$ cada 2 a 4 semanas si es necesario, para alcanzar el objetivo de la uricemia. Si el objetivo no puede ser alcanzado con una dosis apropiada de alopurinol, este debe ser cambiado a febuxostat 0 un uricosúrico, 0 ser combinado con un uricosúrico. Febuxostat 0 un uricosúrico también se indican si el alopurinol no es bien tolerado.

En pacientes con insuficiencia renal, la dosis máxima de alopurinol debe ajustarse a la depuración de creatinina. Si no se puede alcanzar el objetivo de AU con esta dosis, el tratamiento debe cambiarse a febuxostat 0 indicar benzbromarona con 0 sin alopurinol, excepto en pacientes con una tasa calculada de filtrado glomerular $<30 \mathrm{ml} / \mathrm{min}$.

En pacientes con gota crónica debilitante y mala calidad de vida, en quienes no se puede alcanzar el objetivo de AU con cualquier otro fármaco disponible en la dosis máxima (incluidas las combinaciones), se indica la pegloticasa.

Cuando la gota se produce en un paciente que recibe diuréticos tiazídicos 0 de asa, se debe sustituir el diurético de ser posible; para la hipertensión considerar losartán o bloqueadores de los canales de calcio; para la hiperlipidemia, considerar una estatina o fenofibrato.

A (tratamiento precoz)

$\mathrm{D}$ (resto de la recomendación)

A (colchicina, AINE, corticoides orales), C (inyección local de corticoides)

A (canakinumab), C (ankinra) 
Figura 1. Algoritmo de manejo de los ataques agudos de gota

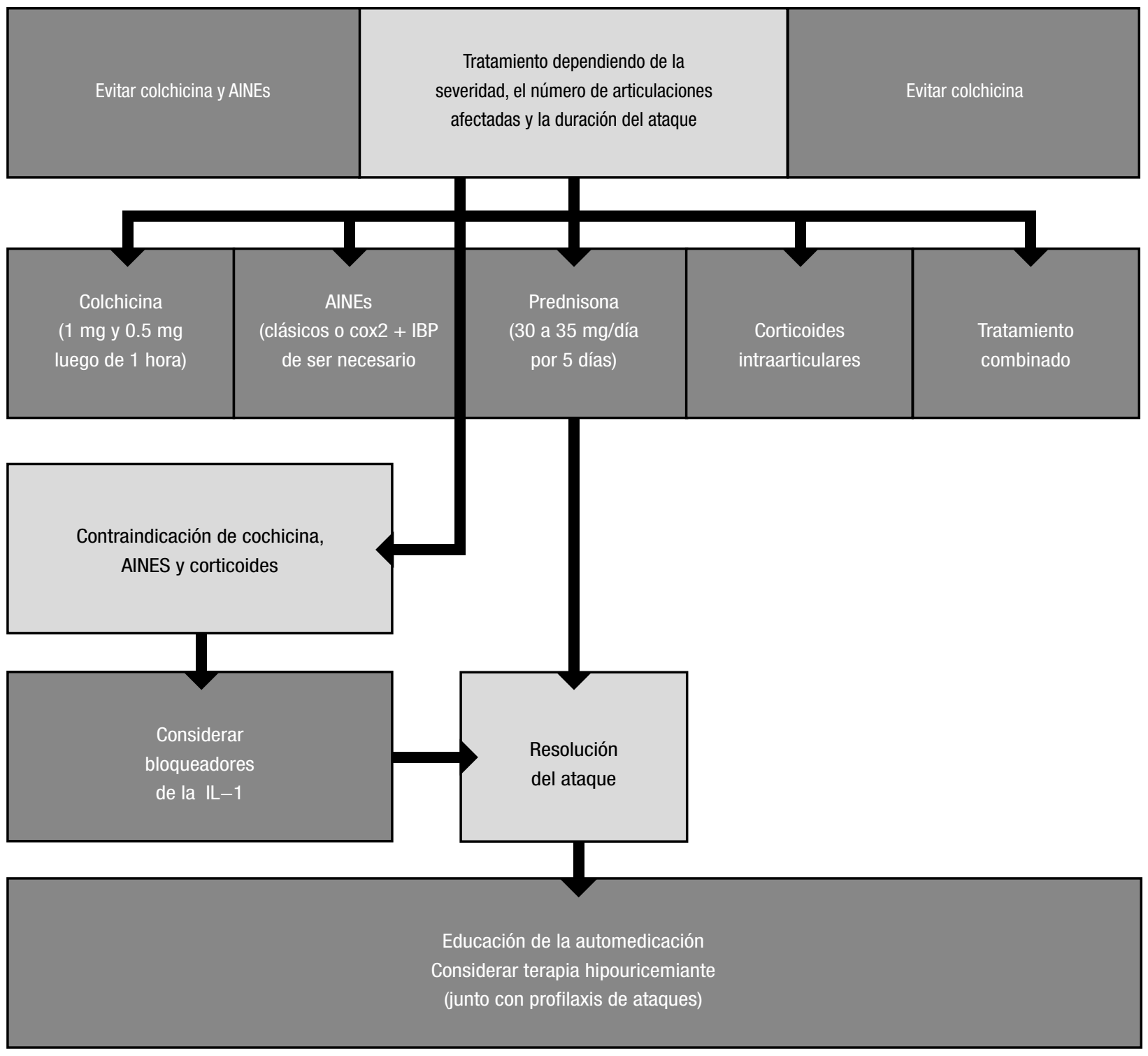

Adaptado del artículo original. 


\section{DETERMINAR VALOR OBJETIVO DEL ÁCIDO ÚRICO}

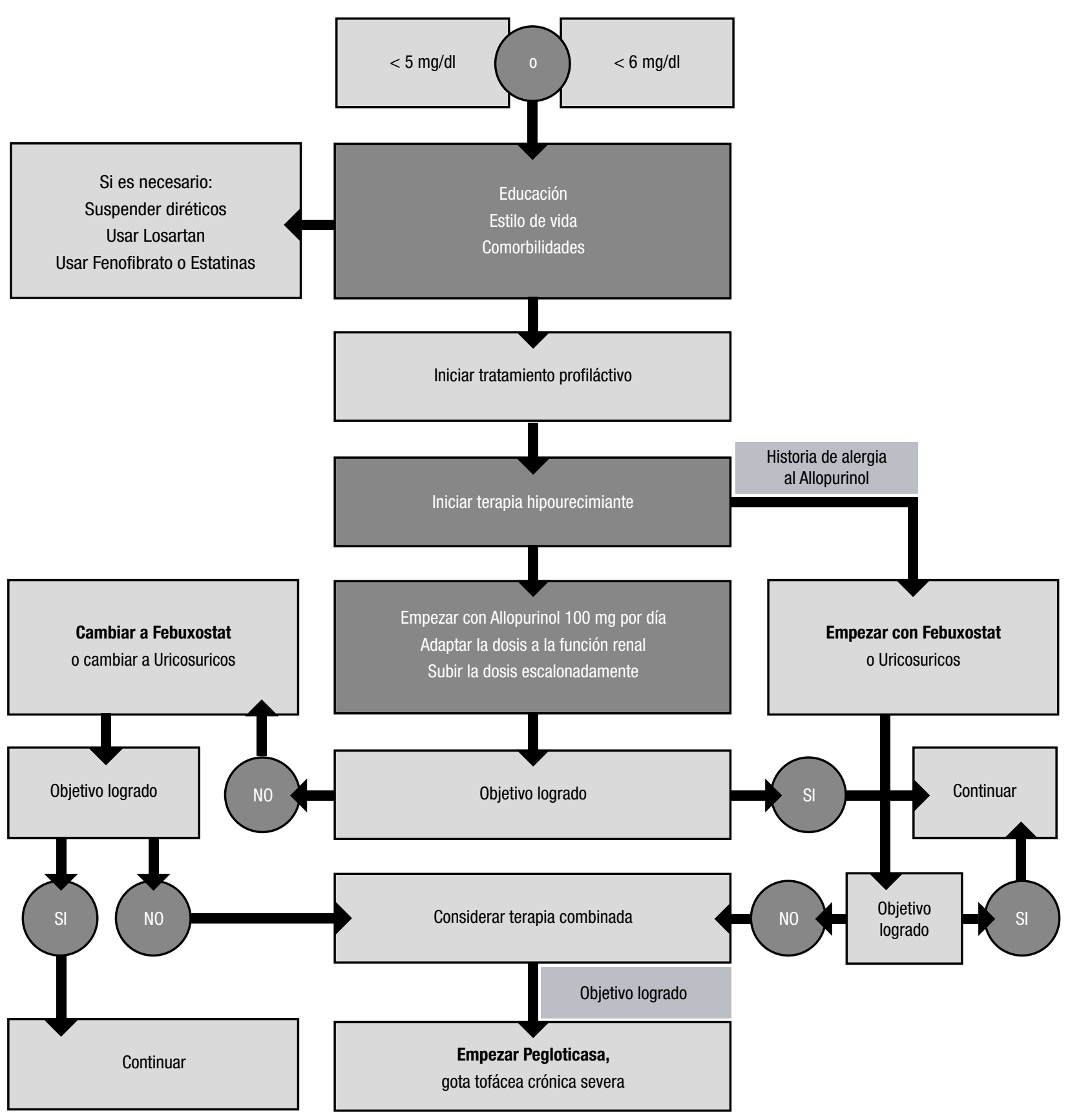

Adaptado del artículo original.

Fuente de financiamiento:

no refiere financiamiento para el desarrollo de las recomendaciones. Se explicita que varios de los autores han recibido aportes económicos de distintas entidades de la industria farmacéutica durante su trayectoria profesional. 


\section{Comentario}

Cabe resaltar algunos conceptos importantes y novedosos:

Los médicos de familia y/o médicos generalistas son propuestos como fundamentales en el tratamiento de la gota, principalmente en los ataques agudos.

Actualmente se cuenta con un mayor número de fármacos para el tratamiento de la gota, tanto con la aprobación del febuxostat como de la pegloticasa, la demostración de la eficacia de los bloqueadores de IL-1 para tratar los ataques agudos y la aparición de nuevas terapias hipouricemiantes.

$\mathrm{Se}$ recomienda iniciar el tratamiento hipouricemiante con bajas dosis de alopurinol y aumentar lentamente la dosis, ya que probablemente esto produzca menor cantidad de episodios de ataques de gota aguda al principio del tratamiento y podría mejorar la adherencia al tratamiento.

La otra novedad es la recomendación de bloqueo de IL-1 en pacientes con ataques recurrentes y mal controlados. Debido a problemas de costos y el posible riesgo de infecciones asociadas con los bloqueadores de IL-1, el grupo de trabajo recomienda su uso en pacientes con contraindicaciones a la colchicina, AINE y corticosteroides.

Finalmente, se hace especial hincapié en la necesidad de educación del paciente acerca de su enfermedad, como así también promover tener un "fármaco en el bolsillo" para el tratamiento rápido de los ataques agudos. El grupo de trabajo es convincente respecto a que los pacientes deben participar plenamente del manejo de su enfermedad.

\section{Conclusiones del comentador}

A pesar de todos los conocimientos sobre las bases fisiopatológicas de la enfermedad y el desarrollo de nuevos fármacos, las tasas de hospitalización primaria por gota aumentaron sustancialmente durante casi dos décadas en los Estados Unidos, mientras que, por ejemplo, las de la artritis reumatoide disminuyeron. Esto podría estar reflejando por un lado el cuidado y la atención subóptima recibida por estos pacientes y por otro el incremento de su prevalencia. Todo esto sugiere la necesidad de mejorar la atención y la prevención en los pacientes con riesgo de desarrollar gota y la atención en aquellos con diagnóstico de esta enfermedad ${ }^{5}$. El hecho de adherirse a las recomendaciones de expertos y sociedades científicas internacionales, en este caso sobre el manejo de la gota, podría ayudar a mejorar la progresión de la enfermedad, la morbimortalidad asociada y sus consecuencias desde el punto de vista funcional.

Santiago Ruta [Sección Reumatología - Servicio de Clínica Médica del Hospital Italiano de Buenos Aires. santiago.ruta@hospitalitaliano.org.ar]

Ruta S. Recomendaciones de la Liga Europea contra el Reumatismo para el manejo de la gota. Evid Act Pract Ambul. 2018;21(1):24-28. Comentado de: Richette P, y col. 2016 updated EULAR evidence-based recommendations for the management of gout. Ann Rheum Dis. 2017 Jan;76(1):2942. PMID: 27457514.

\section{Referencias Bibliográficas}

1.Neogi T. Clinical practice. Gout. N Engl J Med. 2011;364(5):443-52.

2.Terkeltaub RA. Clinical practice. Gout. N Engl J Med. 2003;349(17):1647-55.

3.Neogi T, y col. 2015 Gout classification criteria: an American College of Rheumatology/European League Against Rheumatism collaborative initiative. Ann Rheum Dis. 2015;74(10):1789-98.

4.Neogi T, y col. 2015 Gout Classification Criteria: an American College of Rheumatology/European League Against Rheumatism collaborative initiative. Arthritis Rheumatol. 4.Neogi T, y col. 2015 (2015;67(10):2557-68.
2.

5.Lim SY, y col. Trends in Gout and Rheumatoid Arthritis Hospitalizations in the United States, 1993-2011. JAMA. 2016;315(21):2345-7.

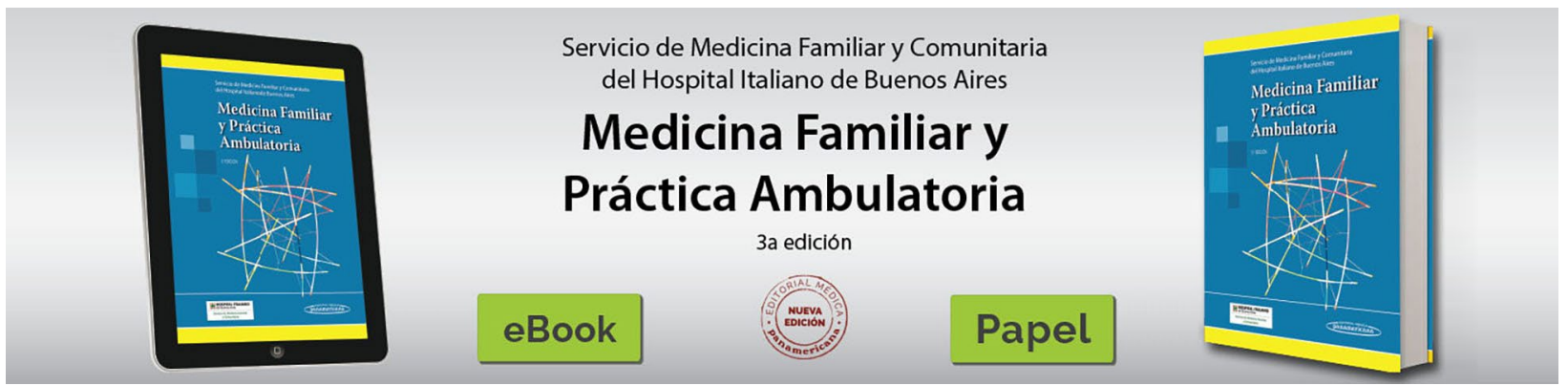

